\title{
Mechanisms of Atrial Fibrillation and Their Impact on Strategies for Catheter Ablation
}

\author{
Norbert Guettler, ${ }^{1,2}$ Edward Nicol, ${ }^{3}$ Joern Schmitt ${ }^{4}$ and Kim Rajappan ${ }^{5}$ \\ 1. Internal Medicine and Cardiology, German Air Force Center for Aerospace Medicine, Fuerstenfeldbruck, Germany; 2. Central Military Hospital, \\ Koblenz, Germany; 3. Department of Cardiology, Royal Brompton Hospital, London, UK; 4. Department of Cardiology, University Hospital Giessen, \\ Giessen, Germany; 5. Cardiac Department, John Radcliffe Hospital, Oxford University Hospitals NHS Foundation Trust, Oxford, UK
}

DOI: https://doi.org/10.17925/EJAE.2018.4.2.56

\begin{abstract}
A trial fibrillation (AF) is the most common sustained arrhythmia, and is ubiquitous in clinical practice. The underlying mechanisms of initiation and maintenance of AF are complex and not completely understood. This knowledge, however, is fundamental for the development of treatment strategies for AF. Within the last 20 years, catheter ablation has played an increasing role as a rhythm control therapy. Based on diverse models for the initiation and maintenance of AF, various ablation strategies have been proposed. The cornerstone of AF ablation has been pulmonary vein isolation (PVI). In persistent AF, however, $\mathrm{PVI}$ alone is often not sufficient. This may be because of the structural remodelling of the atria leading to dilation and fibrosis amongst other factors. The optimal strategy for substrate modification, however, is still a matter of investigation. Current studies are concentrating on the ablation of fibrotic areas, especially in the left atrium, either detected by delayed enhancement magnetic resonance imaging or by identification of low-voltage areas as a surrogate marker. The second intensely evaluated strategy is the localisation and ablation of rotational activity. Many further randomised controlled trials will likely be needed to determine the optimal ablation strategy for individual patients.
\end{abstract}

\section{Keywords}

Atrial fibrillation, electrophysiological mechanisms, catheter ablation, atrial remodelling, pulmonary vein isolation, atrial substrate modification

Disclosures: Norbert Guettler, Edward Nicol, Joern Schmitt and Kim Rajappan have nothing to declare in relation to this article.

Review Process: Double-blind peer review.

Compliance with Ethics: This study involves a review of the literature and did not involve any studies with human or animal subjects performed by any of the authors.

Authorship: All named authors meet the criteria of the International Committee of Medical Journal Editors for authorship for this manuscript, take responsibility for the integrity of the work as a whole and have given final approval for the version to be published.

Open Access: This article is published under the Creative Commons Attribution Noncommercial License, which permits any non-commercial use, distribution, adaptation and reproduction provided the original author(s) and source are given appropriate credit. () The Authors 2018

Received: 21 September 2018

Accepted: 19 November 2018

Citation: European Journal of Arrhythmia

\& Electrophysiology. 2018;4(2):56-64

Corresponding Author: Norbert Guettler

German Air Force Center for Aerospace

Medicine, Strasse der Luftwaffe 322, 82256

Fuerstenfeldbruck, Germany. E: guettlern@aol.com

Support: No funding was received in

the publication of this article.
Atrial fibrillation (AF) is one of the most frequent arrhythmias worldwide, and, despite progress in the management of patients with $A F$, remains one of the major causes of stroke, heart failure, sudden death, and cardiovascular morbidity. ${ }^{1}$ The incidence and prevalence of AF is still increasing with higher numbers in developed countries, which is probably caused by better detection of silent $A F$, increasing population age, and increasing prevalence of predisposing factors. ${ }^{2-4}$ For 2030, a prevalence of 14-17 million patients with AF is estimated for the European Union, with $120,000-215,000$ newly diagnosed patients per year. ${ }^{5-7}$ Some of the predisposing factors for AF are increasing age, hypertension, heart failure, coronary artery disease, valvular heart disease, obesity, diabetes mellitus, hyperthyroidism, obstructive sleep apnoea and chronic kidney disease. ${ }^{7-12}$ $\mathrm{AF}$ is independently associated with a twofold increased risk of all-cause mortality in women and a 1.5-fold increase in men..$^{13-15}$ While the risk of death due to stroke can effectively be mitigated by anticoagulation or left atrial appendage occlusion, death due to heart failure or subsequent cardiac and extra cardiac complications remains a problem even in patients treated according to current scientific evidence. Many patients suffer from relevant symptoms ranging from palpitations to anxiety and dyspnoea. The mechanisms of AF are still not completely understood; however, within recent years, considerable progress has been made evaluating the genetic predisposition of $A F$, the remodelling of atrial structure and ion channel function, and electrophysiological mechanisms of AF. The latter have influenced strategies of catheter ablation, which has become increasingly important as a rhythm control therapy, and which has been established as an option of first-line therapy in selected patients with AF.

In this article, recent progress in understanding the pathophysiology of AF will be summarised with an emphasis on electrophysiological mechanisms and their influence on catheter ablation strategies of AF will be described.

\section{Structural remodelling of the atria}

AF can be classified as:

- paroxysmal AF, with spontaneously terminating episodes of not more than 7 days;

- persistent $A F$, with episodes of more than 7 days or the requirement of cardioversion; and

- permanent AF, which is accepted as a constant arrhythmia without any attempts to restore or maintain sinus rhythm.

Persistent AF with a continuous episode of more than 1 year is called long-standing persistent AF. 
The degree of structural remodelling of the atria does not necessarily correlate with this classification. The main changes seen are progressive dilation and fibrosis of the atria. Remodelling can be caused by external stressors like structural heart disease, hypertension, and possibly diabetes, but also by AF itself. Activation of fibroblasts leads to fibrosis, ${ }^{16,17}$ but inflammatory infiltrates, fatty infiltration, myocyte hypertrophy, necrosis, and amyloidosis are also found in patients with predisposing factors leading to $A F^{18-22}$ Structural remodelling of the atria results in electrical dissociation between muscle bundles and local conduction heterogeneities leading to re-entry and perpetuating of the arrhythmia. ${ }^{23}$

\section{Electrophysiological mechanisms of atrial fibrillation}

There are two major principles which have gained acceptance; firstly, factors triggering AF and secondly, factors that perpetuate AF.

\section{Focal initiation of atrial fibrillation}

In 1998, Michel Haïssaguerre and co-workers published their fundamental observation that pulmonary veins (PVS) are an important source of ectopic beats, initiating frequent paroxysms of $\mathrm{AF}^{24}$ They described that radiofrequency (RF) ablation of such drivers can be used to successfully treat AF. Such rapidly firing foci were mainly localised in the myocardial sleeves, muscle fibers near the ostia of the PV. The mechanism of focal activity might involve both triggered activity and localised re-entry. ${ }^{25,26}$ Atrial muscle fibres are oriented in different directions. Abrupt changes in fibre orientation due to mechanical factors can favour re-entry. Triggered activity and abnormal automaticity, the second mechanism of focal activity in the PVs, promote rapid focal firing. Although the PVs are the most common sites for ectopic focal triggers, there are also non-PV triggers arising from different parts of the atria. ${ }^{27}$

\section{Maintenance of atrial fibrillation The multiple wavelets hypothesis}

In 1959, Moe and Abildskov described a multiple wavelet hypothesis. ${ }^{28}$ According to this theory, these wavelets can sustain AF, as long as the number of wavefronts does not decline below a critical level in an appropriate atrial substrate concerning the combination of atrial size and mass, conduction velocity and tissue refractory period. This hypothesis was supported by a computer model of AF in which propagation of multiple atrial impulses demonstrated self-perpetuating activity with many similarities to clinical AF in humans. ${ }^{29}$ The authors observed that a decline of the number of wavelets below a critical value would lead to termination of AF. Allessie et al. supported this model by providing experimental evidence from a canine heart model in 1985. ${ }^{30}$ Based on this theory, Cox et al. mapped multiple re-entrant atrial wavefronts during AF episodes in humans. This was the basis for the development of the surgical maze procedure, creating small electrically isolated atrial compartments by cutting and sewing atrial tissue to prevent sustained re-entry. ${ }^{31}$ Meanwhile it has been accepted that separate mechanisms may be responsible for triggering and sustaining AF.32 Localised sources, for example foci in the PV, can initiate AF. AF maintenance, however, probably involves some form of re-entrant activity caused by a wavebreak of the main re-entrant wavefront into multiple chaotic daughter wavelets because of inhomogeneity in atrial structure, refractoriness and conduction velocity. ${ }^{33}$

\section{Functional re-entry and the leading circle model}

The leading circle model described by Allessie et al. can be regarded as the simplest form of functional re-entry. ${ }^{34}$ In this model, circus movement of a unidirectional wavefront results in constant centripetal activation of the center rendering it continuously refractory. This refractory area in the

\section{Figure 1: Schematic of a rotor or spiral wave}

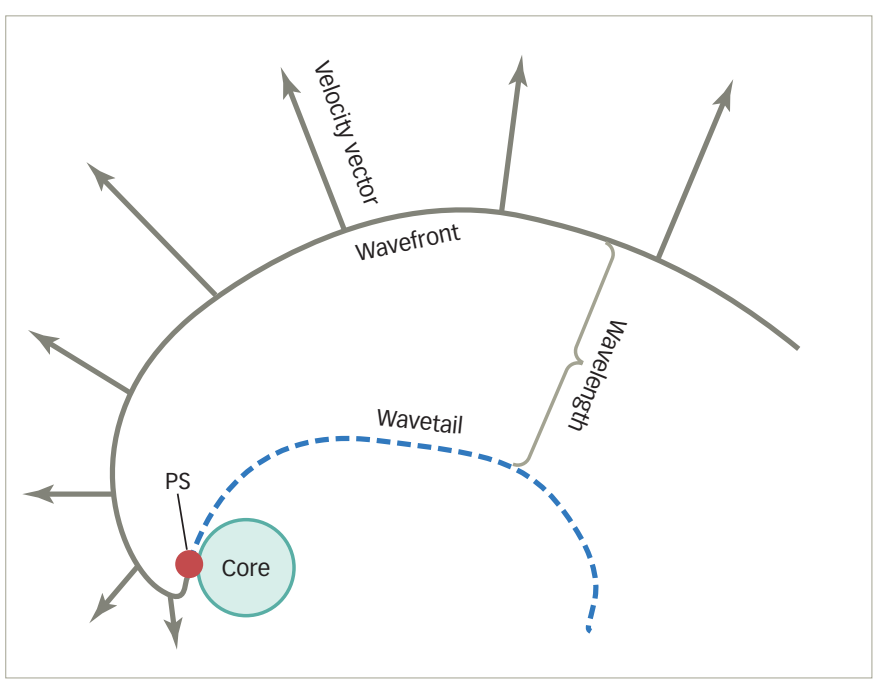

Wavefront and wavetail meet at a focal point called phase singularity. Near the PS, the wavefront shows the highest curvature and therefore the slowest conduction velocity. The tissue core in the center of the rotor cannot be invaded by the wavefront and is effectively unexcitable. However, the core is not truly refractory so that the rotor can move through space.

$P S=$ phase singularity.

center of the circuit forms a functional barrier for electrical conduction which can sustain re-entry like a fixed anatomic barrier, such as a scar. In the leading circle model, unidirectional block in tissue allows an impulse to start circus movement in one direction, with the impulse simultaneously spreading radially outwards to activate the adjacent myocardium and radially inwards towards the center of the circuit. The leading circle is the smallest circuit that can sustain re-entry. If the circuit was smaller, the wavefront would hit refractory tissue and therefore block and terminate. The smallest possible circuit rotates with the highest frequency and will overdrive and suppress all larger circuits. A core of refractory tissue will remain in the center of this circuit. 35

The number of re-entrant circuits that can be sustained depends on wavelength and atrial size. Large atria and small re-entrant circuits allow multiple re-entrant circuits, increasing the probability of sustaining AF. In contrast, in small atria or those surgically partitioned after maze procedure with large re-entrant circuits, maintenance of re-entry and AF is unlikely. ${ }^{36}$

Recent experimental data, however, have suggested that despite the importance of functional re-entry in initiating and sustaining $A F$, it is significantly more complex than described in the leading circle model with multiple simultaneous wavelets.

\section{Functional re-entry due to rotors or spiral waves}

Based on several theoretical studies, it was postulated that rotors generating spiral waves, rather than multiple randomly propagating wavelets, could be the underlying cause of AF. In 1990, Davidenko et al. first demonstrated such a spiral wave in an isolated sheep ventricular muscle slice. ${ }^{37}$ Rotors, or spiral waves, describe a specific type of functional re-entry. The wavefront has a curved or spiral form (Figure 1) instead of being circular. Wavefront and wavetail meet at a focal point called phase singularity (PS). ${ }^{32}$ Unlike in the leading circle model, the wavefront velocity in a rotor is not constant. The wavefront near the PS is the region of the highest curvature and therefore of the slowest wavefront conduction velocity. This has the effect that a tissue core in the centre of the rotor cannot be invaded by 
the wavefront and is effectively unexcitable. But unlike the leading circle model, the core is not truly refractory. Because of this difference, rotors can move through space, in contrast to re-entrant circuits in the leading circle model. The variable curvature of a rotor establishes a gradient of conduction velocity and gradients of action potential duration and wavelength which rise with increasing distance from the PS. This results in a gradient of excitable gap and heterogeneous conduction properties following the spiral shape of the rotor and influencing its behaviour. ${ }^{35,38}$ Rotors form when a wavefront interacts with a barrier which can either be structural, like a scar, or functional, like a myocardial electrical inhomogeneity or anisotropy. When the wavefront passes through a barrier, it can bend and break into two daughter wavelets by a process called vortex shedding. These daughter wavelets can rotate around a PS and form stable rotors in a certain place, like around a PV or in areas of heterogeneous atrial tissue. ${ }^{39}$ When the rotating wavefront spreads away from PS and core, it can interact with other areas of functional inhomogeneity and induce disorganised fibrillatory waves which then induce the chaotic atrial activation associated with $\mathrm{AF}^{40}$

There has been evidence for rotors and other high frequency sources as drivers of AF from some mainly experimental studies. ${ }^{41-46}$ The strongest clinical evidence for high-frequency rotors and focal drivers and their critical role in maintaining human AF, however, comes from the work by Narayan et al. about the mapping and ablation of focal impulses and rotors. ${ }^{47}$

\section{Complex fractionated atrial electrograms}

complex fractionated atrial electrograms (CFAES), and their role in the maintenance of $A F$, have been discussed for many years. They can be caused by multiple mechanisms, but in general represent areas of the myocardium with separated or disorganised myocardial fibres causing slowed, dyssynchronous, and/or anisotropic local conduction. ${ }^{48,49}$ In human AF it has been proposed that CFAEs may be caused by local collision of multiple wavelets, zones of slow conduction, local re-entry, areas near high frequency sites where wavebreak and fibrillatory conduction occur or by direct autonomic innervations. ${ }^{49,50}$ It has been assumed that these fractionated sites are important in sustaining $A F^{51}$ and that targeting

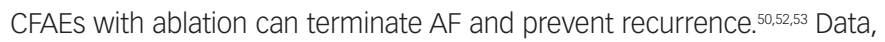
however, are rather weak and contradictory. A meta-analysis evaluated the outcomes in 622 patients across seven trials comparing PV isolation (PVI) alone with PVI plus CFAE ablation. In conclusion, the addition of CFAE ablation to PVI resulted in only a modest increase in maintenance of sinus rhythm without antiarrhythmic drugs (relative risk [RR] 1.17, 95\% confidence interval $[\mathrm{Cl}]$ 1.03-1.33, $\mathrm{p}=0.019$ ), and in subgroup analysis only patients with non-paroxysmal AF had any benefit (RR $1.35,95 \% \mathrm{Cl}$ 1.04-1.75, $\mathrm{p}=0.022){ }^{54}$ Another more recent meta-analysis confirmed these results; CFAE ablation did not significantly reduce AF recurrence. ${ }^{55}$ In contrast to initial theories concerning the relation of CFAES to AF, CFAES seem to be non-specific electrophysiological manifestations which are unlikely to be important drivers of AF. The occasional success of CFAE ablation in persistent AF can probably be explained by coincidental ablation of re-entrant circuits and AF drivers. CFAE ablation has minimal effect on patient outcomes, but can increase procedure times and complication rates. ${ }^{32}$

\section{The influence of autonomic nervous system and ganglionated plexi on atrial fibrillation}

The autonomic nervous system is likely to play a role in clinical $A F^{56-58}$ Ganglionated plexi (GP) are epicardial networks of autonomic nerves near the junctions between the PV and the left atrium (LA). ${ }^{59,60}$ Autonomic signals to the heart via GP can alter atrial refractory periods, ${ }^{61,62}$ increase
Figure 2: Structure and mechanisms of atrial fibrillation

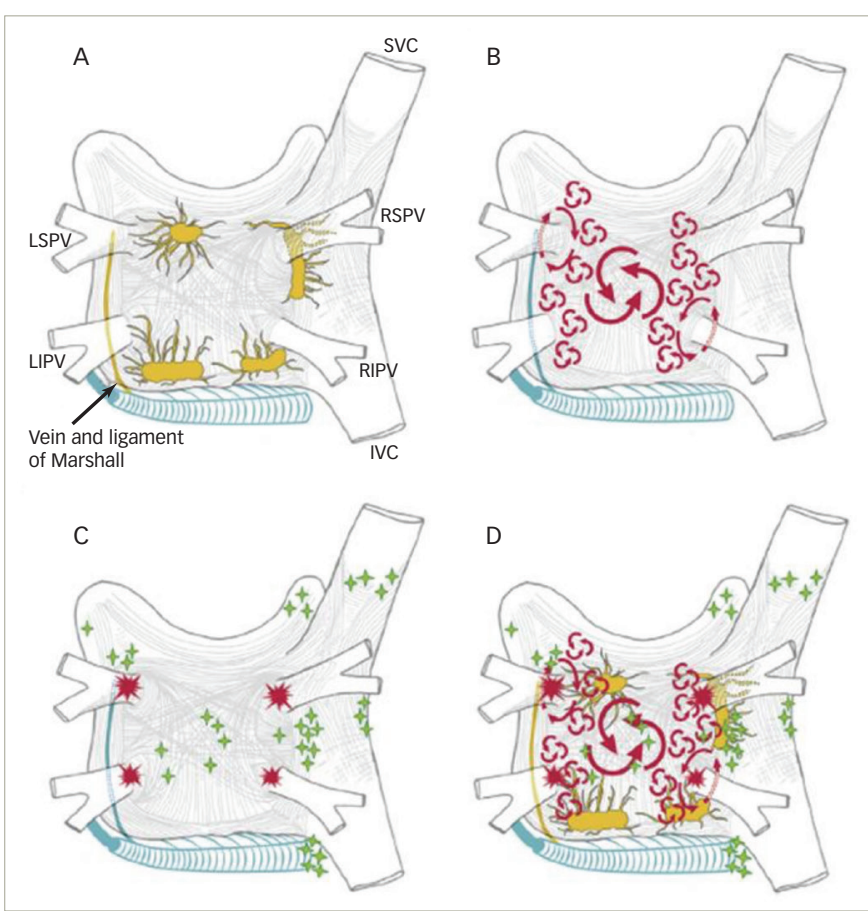

A: Schematic drawing of the left and right atria as viewed from the posterior perspective. The extension of muscular fibers on to the PVS can be appreciated. Shown in yellow are the five major left atrial autonomic GP and axons (superior left $G P$, inferior left $G P$, anterior right GP, inferior right $G P$, and ligament of Marshall). Shown in blue is the coronary sinus, which is enveloped by muscular fibers that have connections to the atria. Also shown in blue is the vein and ligament of Marshall, which travels from the coronary sinus to the region between the left superior PV and the left atrial appendage. B: The large and small re-entrant wavelets that play a role in initiating and sustaining AF. C: The common locations of PV (red) and also the common sites of origin of non-PV triggers (shown in green). D: Composite of the anatomic and arrhythmic mechanisms of AF. AF = atrial fibrillation; $G P=$ ganglionated plexi; $I V C=$ inferior vena cava; $L I P V=$ left inferior pulmonary vein; $L S P V=$ left superior pulmonary vein; $P V=$ pulmonary vein; $R I P V=$ right inferior pulmonary vein; $R S P V=$ right superior pulmonary vein; $S V C=$ superior vena cava.

Reprinted from Journal of Arrhythmia, vol 33, Calkins et al. 2017 HRS/EHRA/ECAS/ APHRS/SOLAECE expert consensus statement on catheter and surgical ablation of atrial fibrillation: Executive summary. Pages 369-409 (2017), ${ }^{68}$ with permission from Elsevier.

the frequency of triggered atrial premature beats that can subsequently initiate $\mathrm{AF}^{63}$ and increase the vulnerability of atrial tissue to the induction of AF. 61,64,65 Animal studies have demonstrated that neuronal/autonomic blockade with drugs or GP ablation can prevent AF induction.66,67

As GP are located near the PV, it can be assumed that conventional PVI often coincidently eliminates these areas. It has been discussed that this could contribute to the ablation success by PVI.

\section{The impact of atrial fibrillation mechanisms on mapping and ablation strategies}

The described mechanisms of AF have influenced mapping and ablation strategies. For paroxysmal AF, antrum isolation of the PV is usually sufficient, while strategies for catheter ablation of persistent AF are still debated. Structure and mechanisms of AF are summarised in Figure $2{ }^{68} \mathrm{AF}$ ablation strategies, underlying mechanisms of $\mathrm{AF}$, and their importance for clinical practice are summarised in Table 1.24,4,53,54,69-95

\section{Factors influencing the outcome of catheter ablation for atrial fibrillation}

Besides the choice of an appropriate ablation strategy, there are several factors and cardiac diseases that compromise especially the LA and adversely affect the outcome of catheter ablation for AF. 
Table 1: Atrial fibrillation ablation strategies, underlying mechanisms, and role in clinical practice

\begin{tabular}{|c|c|c|c|}
\hline Ablation strategy & Underlying AF mechanism & Role in clinical practice & References \\
\hline Antral isolation of all PVs & $\begin{array}{l}\text { Focal triggering and localised re-entry from } \\
\text { the PVs }\end{array}$ & Primary ablation target in paroxysmal and persistent AF & $24,78,79$ \\
\hline Ablation of non-PV triggers & Focal triggers not located in the PVs & $\begin{array}{l}\text { Persistent and sometimes paroxysmal AF, no routine } \\
\text { technique because of challenging localisation of non-PV } \\
\text { triggers }\end{array}$ & $110-112,114$ \\
\hline Linear lesions & Multiple wavelet and leading circle models & No longer used routinely because of poor study results & 98-114 \\
\hline CFAES & Unclear & No longer used routinely because of poor study results & $53,54,98-114$ \\
\hline Ablation of fibrotic areas & $\begin{array}{l}\text { Atrial structural remodelling with fibrosis and } \\
\text { scar formation }\end{array}$ & Promising strategy of substrate modification in persistent AF & 105-109 \\
\hline Ablation of rotational activity & $\begin{array}{l}\text { Functional re-entry due to rotors and spiral } \\
\text { waves }\end{array}$ & $\begin{array}{l}\text { Persistent AF, contradictory study results, large randomised } \\
\text { studies needed }\end{array}$ & 47,122-124 \\
\hline Posterior wall isolation & $\begin{array}{l}\text { Non-PV triggers and fibrotic areas at posterior } \\
\text { wall of LA }\end{array}$ & $\begin{array}{l}\text { Persistent AF, contradictory results, durable lesions probably } \\
\text { crucial, further studies needed }\end{array}$ & $112,119-121$ \\
\hline Ablation of ganglionated plexi & $\begin{array}{l}\text { Influence of the autonomic nervous system } \\
\text { on AF }\end{array}$ & $\begin{array}{l}\text { Persistent AF, only a few small studies conducted, further } \\
\text { study results needed }\end{array}$ & 125,127 \\
\hline
\end{tabular}

$A F=$ atrial fibrillation; $C F A E=$ complex fractionated atrial electrogram; $L A=$ left atrium; $P V=$ pulmonary vein .

It has been known for a long time that ablation success significantly depends on LA size. ${ }^{96,97}$ Other cardiac conditions with a negative influence on ablation success are left ventricular dysfunction, 98,99 hypertrophic cardiomyopathy ${ }^{100}$ and mitral regurgitation. ${ }^{101}$

\section{Isolation of the pulmonary veins}

The observation of Haïssaguerre et al. that ectopic beats from the PV can initiate AF led to the cornerstone of AF ablation according to current guidelines, ${ }^{1}$ namely PVI. As AF trigger sites are often located in the antrum of $\mathrm{PV}, \mathrm{PVI}$ often involves encircling ipsilateral veins together with RF current as a wide area circumferential ablation. ${ }^{102}$ Figure 3 shows voltage maps of the LA before and after wide area circumferential ablation. Focal ablation inside the PV has been abandoned because of the risk of PV stenoses. Cryoballoon ablation and laser ablation are alternative energy sources to RF ablation. In the Fire and Ice Trial, cryoballoon ablation has been shown to be non-inferior to RF ablation. ${ }^{103}$ Recently, a meta-analysis revealed similar overall success rates at 12 months and comparable fluoroscopy and procedural times as well as long-term complications between patients undergoing cryoballoon and RF ablation for AF. ${ }^{104}$ The authors concluded that operators should chose AF ablation technology based on patient-specific features and preferences as well as operator experience and preference.

The strategy of PVI, however, is much more effective in paroxysmal AF than it is in persistent AF. Miyazaki et al. reported that about $70 \%$ of patients with paroxysmal AF were in sinus rhythm without any antiarrhythmic drugs with a mean follow-up of 2.5 years after a single PVI procedure. ${ }^{69}$ AF recurrence after PVI was mainly caused by PV reconnection. ${ }^{70}$ It was shown, however, that $\mathrm{PV}$ reconnection after $\mathrm{PVI}$ was present in $90 \%$ of patients with paroxysmal AF without AF recurrence. ${ }^{105}$ Therefore, sustained $\mathrm{PVI}$ might not be required to prevent clinical recurrence in patients with paroxysmal AF. In paroxysmal AF triggers are probably limited to certain areas, and reconnection of PVS without such triggers may not cause AF recurrence. ${ }^{106}$ It was reported that approximately $70 \%$ of patients with paroxysmal AF originating from the superior vena cava remained in sinus rhythm after isolation of the vena cava without accompanying PVI after a follow-up period of 5 years. ${ }^{107}$ But as exact localisation of AF triggers is often difficult, empiric durable antrum isolation of all PV should be performed to minimise AF recurrence.
Figure 3: Left atrium voltage maps before and after wide area circumferential ablation for pulmonary vein isolation

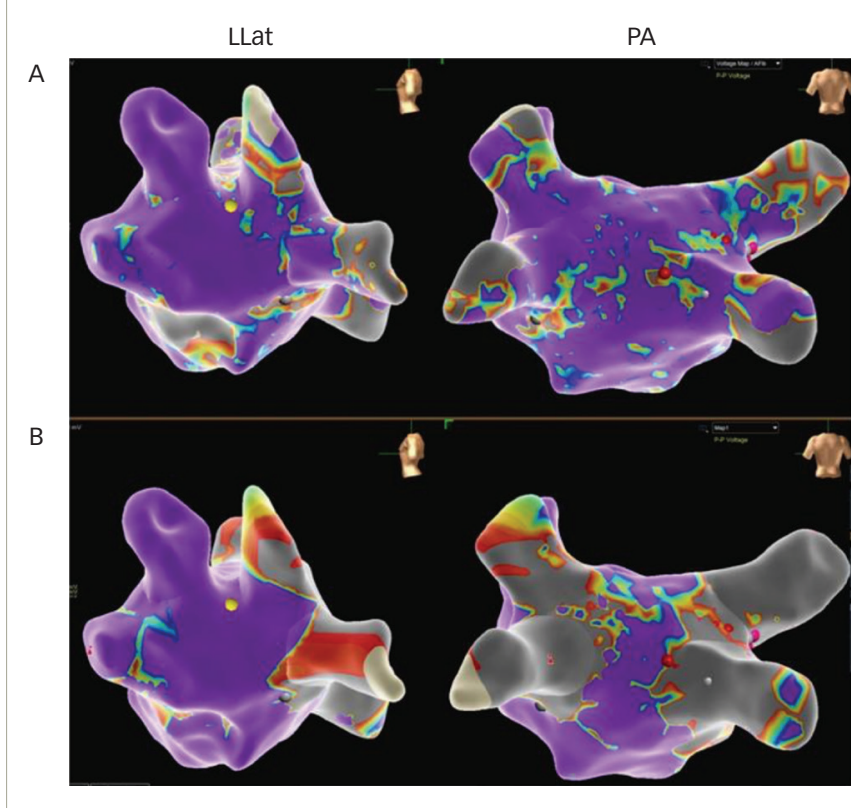

Left atrium voltage maps before (A) and after (B) wide area circumferential ablation for pulmonary vein isolation using the EnSite Precision ${ }^{\mathrm{TM}}$ cardiac mapping system (Abbott, Chicago, IL, US). The purple represents normal voltage and the grey represents low voltage or scar. Note the distal part of the pulmonary veins is grey before ablation where the muscle sleeves end. After ablation, the grey begins distal of the ablation lesion. The images on the left are left lateral views and the ones on the right are posterior-anterior views.

$L L a t=$ left lateral views; PA = posterior-anterior views.

In persistent AF, efficacy of PVI has been limited, ${ }^{108,109}$ and in many patients $\mathrm{PV}$ reconnection was reported to be the cause of AF recurrence. ${ }^{110}$ In persistent $A F$, there may be sites of $A F$ initiation and maintenance in all four PVs, so that reconnection even in one PV may result in $\mathrm{AF}$ recurrence. Thus, permanent electrical isolation of all PVs is much more crucial in persistent than in paroxysmal $\mathrm{AF}^{106}$ This can be achieved more effectively by using irrigated RF ablation catheters with a contact force (CF) sensor. Using a CF sensor during PVI was reported to correlate with a more positive clinical outcome.111 The TOCCATA (TOuCh+ for CATheter Ablation) study reported that an average $\mathrm{CF}$ of $<10 \mathrm{~g}$ generally led to 
AF recurrence, while maintenance of sinus rhythm could be achieved with an average CF of $>20$ g. ${ }^{111}$ Another study showed that PVI with a CF of 10-20 g significantly reduced AF recurrence in patients with paroxysmal AF.112 The Efficacy Study on Atrial Fibrillation Percutaneous Catheter Ablation with Contact Force Support (EFFICAS I) proposed a target CF of $20 \mathrm{~g}$ and a minimum force-time integral, an index to assess the lesion formation, of $400 \mathrm{~g}$ for each new lesion. ${ }^{113}$ To further improve the prediction of PVI success the lesion size index ${ }^{114}$ and the ablation index $(\mathrm{Al})^{115}$ were defined. But universal consensus has not yet been reached on the values required to create a durable lesion. This may also be influenced by atrial wall thickness. ${ }^{116} \mathrm{~A}$ step towards a standardised protocol for durable PVI is the CLOSE protocol. ${ }^{117}$ It is a point-by-point approach aiming to enclose PVs with contiguous and optimised RF lesions. This protocol was based on a prior study from El Haddad et al. ${ }^{118}$ In this study, 42 CF-guided PVI procedures were analysed with the aim of identifying determinants of acute and late gaps after CF-guided PV encircling. Two independent predictors of durable segments were identified: Al and interlesion distance (ILD). Combining ILD $D_{\max } \leq 6 \mathrm{~mm}$ and $A I_{\min } \geq 400$ (posterior)/550 (anterior) was associated with a $93 \%$ specificity to predict durable segments. Based on these results, the CLOSE protocol ${ }^{117}$ involved creating a nephroid-shaped circle enclosing the veins. Ablation was performed where the atrial myocardium connects to the PV sleeve, and typically with a $>2 \mathrm{~cm}$ distance between the ablated anterior and posterior carina. Lesions were closely spaced with an ILD $D_{\max } \leq 6 \mathrm{~mm}$. Energy of $35 \mathrm{~W}$ was delivered to reach a minimal $\mathrm{Al} \geq 400$ at the posterior wall and $\geq 550$ at the anterior wall. The Al target was reduced to 300 in case of signs of oesophageal injury (pain during local anaesthesia or temperature probe heating during general anaesthesia). The procedural endpoint was not PVI per se but a perfect circle leading invariably to PVI. This CLOSE protocol was evaluated in > 400 patients with paroxysmal AF. A prospective, multi-center, randomised trial is required.

\section{Possible atrial substrate modification strategies for persistent atrial fibrillation ablation}

Whereas paroxysmal AF is predominantly driven by focal activity or local re-entry from the PV, AF-maintaining mechanisms are moving towards the atria and are increasingly based on re-entry substrates in persistent AF. This is caused by several types of atrial remodelling. That means that ablation strategies in persistent AF increasingly must address atrial substrates in addition to the PV. ${ }^{119-122}$

\section{Linear lesions and complex fractionated atrial electrograms ablation}

Former procedures for the ablation of persistent AF often included the creation of linear lesions in the LA and focal ablation of CFAEs in the left or in both atria. The rational for creating linear lesions was mainly imitating the maze procedure described by $\mathrm{Cox}^{31}$ with a percutaneous interventional approach. The procedure has not been standardised, but mostly a roof line and a mitral isthmus line were performed.

Haïssaguerre et al. introduced the stepwise ablation approach consisting of PVI and CFAE and linear lesion formation aiming at AF termination in 2005.71,72 Figure 4 shows a schematic drawing of these ablation strategies. But subsequent large registries reported single-procedure success rates of only $35 \%$ and $48 \%$, respectively, after 12 months follow-up. ${ }^{73,74}$ CHASE-AF (Randomized Catheter Ablation of Persistent Atrial Fibrillation Study), a single-centre randomised trial, investigated the stepwise approach aimed at AF termination versus PVI alone for the ablation of persistent $A F^{75}$ The investigators found no difference in arrhythmia recurrence, but a higher incidence of atrial tachycardia in the stepwise ablation approach group. Accordingly, the Alster-Lost-AF trial (Ablation at
Figure 4: Schematic diagram showing typical lesion patterns
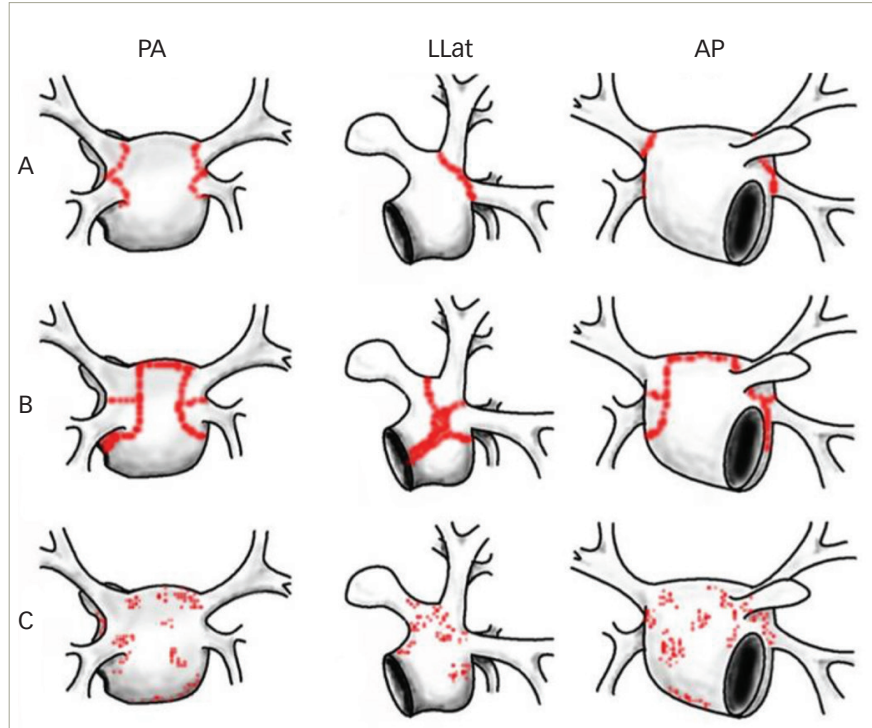

A: Ostial/segmental PVI. B: wide area circumferential ablation with

compartmentalisation/linear ablation (wide area circumferential ablation plus mitral isthmus and roof lines). C: electrogram-guided ablation (CFAE ablation). $A P=$ anterior-posterior views; CFAE = complex fractionated atrial electrogram; $L L a t=$ left lateral views; $P A=$ posterior-anterior views; $P V I=$ pulmonary vein isolation

St. Georg Hospital for Long-Standing Persistent Atrial Fibrillation) could not demonstrate an additional benefit of the stepwise approach aimed at AF termination over PVI alone for persistent and long-standing persistent $A F^{76}$ These results suggested that substrate modification aimed at AF termination is no suitable strategy to reduce AF recurrence in patients with persistent $\mathrm{AF}$.

STAR AF ॥ (Substrate and Trigger Ablation for Reduction of Atrial Fibrillation Trial Part II), a multicenter randomised trial, enrolled 589 patients with persistent AF and compared stand-alone PVI to PVI with additional ablation of CFAEs or linear lesions. ${ }^{77}$ Additional ablation did not reduce AF recurrence. After 18 months, AF recurrence occurred in $41 \%$ of patients with PVI alone, in $51 \%$ of patients with PVI plus CFAE, and in $54 \%$ in patients with PVI plus linear lesions. This well-recognised study influenced the ablation strategy for persistent AF, so that CFAE ablation and linear lesion formation are no longer used routinely by the majority of electrophysiologists. Despite its relatively low efficacy, PVI has become the primary ablation strategy for persistent AF. New strategies for substrate modification are urgently required to further improve the outcome after catheter ablation of persistent AF.

\section{Ablation of fibrotic areas}

Structural remodelling with atrial fibrosis and scarring is a well-recognised factor in the pathogenesis of persistent AF. Left atrial fibrosis and scarring can be detected by delayed enhancement magnetic resonance imaging (DE-MRI). The feasibility of atrial tissue fibrosis estimation by DE-MRI and its association with subsequent AF ablation outcome was examined in the DECAAF study. ${ }^{78}$ The conclusion was that among patients with AF undergoing catheter ablation, atrial tissue fibrosis estimated by DE-MRI was independently associated with likelihood of recurrent arrhythmia. Regions of enhancement on DE-MRI are well correlated with low-voltage regions on electroanatomical maps. ${ }^{79}$ Low-voltage can therefore be regarded as a surrogate marker for atrial fibrosis. Rolf et al. reported that additional ablation for 
low-voltage areas defined as $<0.5 \mathrm{mV}$ recorded during sinus rhythm reduced AF recurrence after PVI. ${ }^{80}$ Methods for detection and ablation of low-voltage areas differed. Yamaguchi et al. demonstrated the effect of regional ablation of low-voltage areas, ${ }^{81}$ whereas Kottkamp et al. proposed circumferential isolation, which they called box isolation of fibrotic areas (BIFA). ${ }^{82}$ Randomised studies are required to confirm the efficacy of this strategy and to compare different approaches.

\section{Ablation of non-pulmonary vein triggers}

Chang et al. reported that patients with non-PV triggers have a worse outcome after AF ablation than those without non-PV triggers. ${ }^{83}$ Catheter ablation of non-PV triggers in addition to PVI improved the outcome in patients with paroxysmal AF. ${ }^{84}$ Santangeli et al. found non-PV triggers in $11 \%$ of patients; they were located in anatomic regions like the inferior mitral annulus, posterior LA, interatrial septum, particularly the fossa ovalis/limbus region, crista terminalis, Eustachian ridge, coronary sinus, and superior vena cava. ${ }^{85}$ Empiric ablation at these sites showed no efficacy in persistent $A F_{\text {, }}^{86}$ but localisation of non-PV triggers is often challenging. Limited ablation of PVS and documented non-PV triggers improved the maintenance of sinus rhythm in patients with persistent and long-standing persistent $A F^{87}$ These results indicate that localisation and ablation of documented non-PV triggers improves the outcome of catheter ablation for persistent AF. So far, there is no standardised protocol for provoking and localising non-PV triggers. In patients without spontaneously firing non-PV triggers, they can, for example, be provoked by an infusion of isoproterenol. ${ }^{23}$ But according to a European survey, only a minority of operators routinely perform provocation and ablation of non-PV triggers in patients with persistent $\mathrm{AF}^{124}$

To estimate the risk of recurrent tachyarrhythmia after AF ablation and to try to define the ablation endpoint, atrial vulnerability has been examined. Atrial refractoriness and atrial conduction time defining a vulnerability index represent latent vulnerability. Furthermore, the inducibility of sustained AF can be assessed by programmed atrial stimulation. AF can be induced, if atrial extrastimuli are delivered during the period of atrial vulnerability, which approximately corresponds to the interval between the atrial relative refractory period and the atrial effective refractory period, also called the latency period. The atrial relative refractory period is usually 10-30 msec greater than the atrial effective refractory period, which mostly ranges from 150 to $260 \mathrm{msec}$. The validity of atrial vulnerability in predicting AF recurrences and defining the ablation endpoint, however, has been shown to be limited. ${ }^{125,126}$

\section{Posterior wall isolation}

The posterior wall of the LA is isolated as a possible site of non-PV triggers or fibrotic areas, ${ }^{85,88}$ but outcome data of routine posterior wall ablation remain contradictory. Incomplete and non-durable posterior wall isolation may have contributed to the reduced efficacy of this method. Bai et al. showed that, in patients with persistent AF, posterior wall isolation in the LA, which was proven by repeat procedure, improved the outcome after ablation. ${ }^{89}$ Another study suggested the usefulness of routine adenosine challenge for dormant posterior wall conduction. ${ }^{90}$ In summary, recent data indicate that durable isolation of the left atrial posterior wall in patients with persistent AF could improve the outcome of catheter ablation.

\section{Ablation for rotational activity}

Rotors have been identified to be important for the maintenance of AF. ${ }^{47}$ Thus, catheter ablation of rotors has recently been evaluated as a possible new ablation strategy for persistent AF. Rotors can be identified by endocardial mapping by the focal impulse and rotor modulation (FIRM) computational mapping system. In the CONFIRM (Conventional Ablation for Atrial Fibrillation with or without Focal Impulse and Rotor Modulation) trial, conventional PVI was compared FIRM-guided ablation, followed by conventional PVI. In a non-randomised manner, 92 patients with drug-refractory paroxysmal (29\%) or persistent (71\%) AF underwent ablation during which 64-pole basket catheters were used to map right and left atria and construct AF activation maps using special computational protocols. In patients with sinus rhythm AF was induced before the mapping procedure. High frequency drivers of AF defined as rotors (areas of spiral or rotational activation) and focal impulses (repetitive focal activation without clear rotation) were identified and targeted with focal ablation followed by conventional PVI. The primary endpoint was acute termination of AF or slowing of the AF cycle length by $\geq 10 \%$. Ninety-seven percent of patients demonstrated rotors or focal impulses, $70 \%$ stable drivers with rotational activity, potentially consistent with rotors. Rotors and focal impulses were more frequent in patients with persistent than with paroxysmal AF, three quarters were found in the $\mathrm{LA}$, and one quarter found in the right atrium. FIRM ablation prior to conventional PVI achieved 56\% termination of AF. In the conventional PVI group, AF termination was achieved in only $9 \%$ of patients. Freedom from AF after a 3-month blanking period was significantly higher in the FIRM-guided ablation group than in the conventional PVI group. Shortcomings of this study were the non-randomised design, the relatively small numbers of patients, and the short follow-up period. The results of this study must be interpreted carefully because of these limitations. Another point of criticism is the mapping with a 64-pole basket catheter with a relatively low resolution. Besides, it might be difficult to get all 64 electrodes in contact with the atrial wall simultaneously. A further mapping technology for rotors is body surface potential mapping, where electrodes from the body surface are projected on thoracic computerised tomography scan images. ${ }^{119}$

Whereas early studies have reported that FIRM-guided ablation in addition to $\mathrm{PVI}$ results in high rates of acute AF termination and long-term freedom from recurrent atrial tachyarrhythmia, ${ }^{47.91}$ more recent studies have failed to confirm these findings. ${ }^{92,93}$ It remains uncertain if $A F$ is truly the result of rotors or if it is secondary to other simpler re-entrant mechanisms. ${ }^{32}$ Multicentre, prospective, randomised studies are necessary to evaluate the efficacy of this new strategy.

\section{Ablation of ganglionated plexi}

Pappone et al. assessed for vagal response (bradycardia, hypotension, heart block or asystole) during ablation and ablated at these sites. Most common areas for vagal response were the junctions between PV and LA, the sites where GP are located. Patients with complete vagal denervation had significantly reduced risks of AF recurrence. ${ }^{94}$ In other studies, high-frequency stimulation has been used to localise GP. Other data, however, have suggested that an anatomic approach should be preferred over high-frequency stimulation or identification of areas with vagal responses. ${ }^{127}$

GP ablation has only been evaluated in a few small studies. ${ }^{95}$ Further trials are needed before it can be adopted into standard practice.

\section{Recent notions about atrial fibrillation mechanisms and their therapeutic implications}

A recent study by de Groot et al. supports the concept that during AF, the endo- and epicardial layers of the atrial wall can be asynchronously activated. ${ }^{228}$ This was evaluated using an endo-epicardial mapping clamp introduced through an intraoperative incision of the right atrium. If patients were in sinus rhythm, AF was induced. The presence of 
dissociated layers of fibrillation waves can stabilise AF, because fibrillation waves dying out in one layer can be replaced by breakthroughs from the opposite side. This transmural conduction of fibrillation waves may explain the persistence of AF and unsuccessful catheter ablations.

Another explanation for unsuccessful ablation attempts could be recent reports about the spatio-temporal instability of AF. In previous studies, target-specific ablation of AF was proposed by directly ablating AF-driving sources exhibiting high-frequency, periodic activity. ${ }^{29}$ These driving sources were regarded as quite stable, so that the non-invasive location of sites with the highest dominant frequency (DF) by panoramic body surface mapping prior to the ablation was considered. Recent studies, however, revealed by means of high-density noncontact mapping that these DF sites were temporally unstable; although, reappearance of DF values occurred at times. It was concluded that targeting sites of peak DF from a single time frame is unlikely to be a reliable ablation strategy. ${ }^{130}$ Mapping results hinted at potentially non-random temporally periodic behaviour of highest DF sites. Another study evaluated the temporal behaviour and consistency of bipolar atrial electrograms (AEG) in human persistent AF. ${ }^{131}$ AEGs were recorded with segment lengths of $2.5,5.0$, and $8.0 \mathrm{~s}$. AEGs with $8.0 \mathrm{~s}$ duration were devided into three consecutive segments of $2.5 \mathrm{~s}$. Forty-three percent of the AEGS remained fractionated for all three of these segments, while nearly $30 \%$ were temporally unstable. This means that a period as short as $2.5 \mathrm{~s}$ for AEG recording, as used in the CARTO ${ }^{\circledR}$ system (Biosense-Webster Inc., Irvine, CA, US) for CFAE mapping, might identify discordant atrial regions as targets for ablation depending on the timing of AEG collection. This could have contributed to the conflicting results of ablation for persistent AF. The detection of a certain spatio-temporal instability of AF will have implications on global or panoramic versus regional mapping strategies for AF ablation.

In recent studies, contact as well as non-contact mapping has been used to detect areas of repetitive high-frequency activation, which possibly interact with high organisation as well as phase mapping targets. In one of these studies, the spatio-temporal association between DF and re-entrant phase activation areas was investigated in persistent AF by means of a non-contact array catheter. ${ }^{132}$ The phase maps presented multiple simultaneous PS points drifting over the LA. Regions of higher PS concentrations showed a degree of colocalisation with DF sites. In $61.8 \%$ of the time window, PS regions were found next to DF regions, in 36.8\% there were overlaps of PS and DF regions. Sites with highest DF showed a greater degree of organisation at their core compared with their periphery. After PVI, PS incidence was reduced over the entire LA, supporting the assumption that PS sites are of some relevance for the atrial substrate. In another study, a novel real-time three-dimensional mapping technique was used to identify repetitive-regular activities (RRas). ${ }^{133}$ Patients were randomised into a group undergoing ablation of RRa followed by modified circumferential PV ablation, and another group assigned to modified circumferential PV ablation alone. Thirty-nine percent of RRas were identified in the PVs, 61\% in non-PV regions. RRa mapping-guided ablation resulted in higher arrhythmia termination rates compared with the conventional approach (61\% versus $30 \%$ ) and had an adjunctive benefit in terms of arrhythmia freedom at 1-year follow-up. Honarbakhsh et al. looked at the identification of focal sources of AF. ${ }^{134}$ They analysed either basket or Penta Ray recordings using CARTOFINDER (Biosense-Webster, Diamond Bar, CA, US) and a defined region of interest algorithm ( $\geq 2$ consecutive focal activations with one electrode leading relative to its neighbours with QS morphology on the unipolar electrogram). Drivers identified as regions of interest were associated with a greater temporal stability, a higher recurrence rate, and a higher number of AF terminations compared to those not identified as a region of interest. This suggests that these sources may be a viable therapeutic target in persistent AF ablation.

All these findings should be confirmed in prospective randomised studies and may influence future ablation strategies for persistent AF.

\section{Conclusion}

Despite the clinical importance of $A F$, the understanding of pathophysiology and mechanisms of this arrhythmia remain incompletely understood. There have been various models proposed for AF mechanisms over time, influencing treatment strategies. Within the last 20 years, catheter ablation of AF has played an emerging role for rhythm control therapy of $A F$, and several ablation strategies have been derived from the different mechanisms of AF proposed. The primary target of AF ablation is the isolation of all PV, in both paroxysmal and persistent AF. In persistent AF, additional substrate modification is often necessary to prevent recurrence of $\mathrm{AF}$ in the structural remodelled atria. Ablation of fibrotic areas in the LA is currently one of the most promising strategies for substrate modification, even though it has not yet been standardised. The first results of the ablation of rotational activity in both atria were impressive, but more recent studies in this field have been contradictory. Large randomised studies are needed for almost all the most modern proposed techniques, whilst historic techniques such as linear lesion formation and CFAE ablation have lost importance in clinical practice because of poor study results.

AF may have multiple, disparate mechanisms in different patients or even in the same patient at different times; therefore, adequate AF ablation, especially for persistent AF, will probably require a patient-tailored approach instead of a 'one size fits all' strategy. $\square$
1. Kirchhof P, Benussi S, Kotecha D, et al. 2016 ESC Guidelines for the management of atrial fibrillation developed in collaboration with EACTS. Eur Heart J. 2016;37:2893-962.

2. Kishore $A$, Vail $A$, Majid A, et al. Detection of atrial fibrillation after ischemic stroke or transient ischemic attack: a systematic review and meta-analysis. Stroke. 2014;45:520-6.

3. Sanna T, Diener HC, CRYSTAL. Cryptogenic stroke and underlying atrial fibrillation. N Eng/ J Med. 2014;370:2478-86.

Schnabel RB, Yin X, Gona P, et al. 50 year trends in atrial fibrillation prevalence, incidence, risk factors, and mortality in the Framingham Heart Study: a cohort study. Lancet. 2015;386:154-62.

5. Colilla S, Crow A, Petkun W, et al. Estimates of current and future incidence and prevalence of atrial fibrillation in the U.S. adult population. Am I Cardiol. 2013;112:1142-7.

6. Krijthe BP, Kunst A, Benjamin EJ, et al. Projections on the number of individuals with atrial fibrillation in the European Union, from 2000 to 2060. Eur Heart J. 2013;34:2746-51.

7. Zoni-Berisso M, Lercari F, Carazza T, et al. Epidemiology of atrial fibrillation: European perspective. Clin Epidemiol. 2014;6:213-20.

8. MCManus DD, Rienstra M, Benjamin EJ. An update on the prognosis of patients with atrial fibrillation. Circulation 2012;126:e143-6

9. Ball J, Carrington MJ, McMurray JJ, et al. Atrial fibrillation: profile and burden of an evolving epidemic in the 21st century. Int $J$ Cardiol. 2013;167:1807-24.

10. Kannel WB, Wolf PA, Benjamin EJ, et al. Prevalence, incidence, prognosis, and predisposing conditions for atrial fibrillation: population-based estimates. Am J Cardiol. 1998;82:2N-9N

11. Oldgren J, Healey JS. RE-LY Atrial Fibrillation Registry Investigators, et al. Variations in cause and management of Investigators, et al. Variations in cause and management of
atrial fibrillation in a prospective registry of 15,400 emergency atrial fibrillation in a prospective registry of 15,400 emergency department patients in 46 countries: the RE-LY Atrial Fibrillation Registry. Circulation. 2014,129.1568-76.

12. Chiang CE, Naditch-Brule L, Murin J, et al. Distribution and risk profile of paroxysmal, persistent, and permanen atrial fibrillation in routine clinical practice: insight from the reallife global survey evaluating patients with atrial fibrillation international registry. Circ Arrhythm Electrophysiol. 2012;5:632-9.

13. Benjamin EJ, Wolf PA, D'Agostino RB, et al. Impact of atrial fibrillation on the risk of death: the Framingham Heart Study. Circulation. 1998;98:946-52.
14. Stewart S, Hart CL, Hole DJ, et al. A population-based study of the longterm risks associated with atrial fibrillation: 20-year follow-up of the Renfrew/Paisley study. Am J Med. 2002;113:359-64.

15. Andersson T, Magnuson A, Bryngelsson IL, et al. All-cause mortality in 272,186 patients hospitalized with incident atrial fibrillation 1995-2008: a Swedish nationwide long-term casecontrol study. Eur Heart J. 2013;34:1061-7.

16. Chimenti C, Russo MA, Carpi A, et al. Histological substrate of human atrial fibrillation. Biomed Pharmacother. 2010;64:177-83.

17. Nguyen BL, Fishbein MC, Chen LS, et al. Histopathological substrate for chronic atrial fibrillation in humans. Heart Rhythm. 2009;6:454-60.

18. Frustaci A, Chimenti C, Bellocci F, et al. Histological substrate of atrial biopsies in patients with lone atrial fibrillation. Circulation. 1997;96:1180-4

19. Venteclef N, Guglielmi V, Balse E, et al. Human epicardial adipose tissue induces fibrosis of the atrial myocardium through the secretion of adipofibrokines. Eur Heart J. 2013;36:795-805a.

20. Rocken C, Peters B, Juenemann G, et al. Atrial amyloidosis: 
an arrhythmogenic substrate for persistent atrial fibrillation. Circulation. 2002;106:2091-7.

21. Schotten U, Ausma J, Stellbrink C, et al. Cellular mechanisms of depressed atrial contractility in patients with chronic atria fibrillation Circulation. 2001:103:691-8

22. Zhang $\mathrm{H}, \mathrm{Li} \perp$, Chen $\mathrm{X}$ et al Association of systemic inflammation score with atrial fibrillation: A case-contro study with propensity score matching. Heart Lung Circ 2018;27:489-96

23. Allessie MA, de Groot NM, Houben RP, et al. Electropathological substrate of long-standing persistent atrial fibrillation in patients with structural heart disease: longitudinal dissociation. Circ Arrhythm Electrophysiol. 2010;3:606-15.

24. Haïssaguerre M, Jaïs $\mathrm{P}$, Shah DC, et al. Spontaneous initiation of atrial fibrillation by ectopic beats originating in the pulmonary veins. N Eng/ J Med. 1998;339:659-66.

25. Patterson $\mathrm{E}$, Jackman WM, Beckman $\mathrm{KJ}$, et al. Spontaneous pulmonary vein firing in man: relationship to tachycardia-pause early afterdepolarizations and triggered arrhythmia in canine pulmonary veins in vitro. $J$ Cardiovasc Electrophysiol. 2007:18:1067-75.

26. Atienza F, Almendral J, Moreno J, et al. Activation of inward rectifier potassium channels accelerates atrial fibrillation in humans: evidence for a reentrant mechanism. Circulation. 2006:114:2434-42.

27. Nabar N, Pathan I. Pathophysiology of atrial fibrillation current concepts. J Assoc Physicians India. 2016;64 (Suppl. 1):11-15.

28. Moe GK, Abildskov JA. Atrial fibrillation as a self-sustaining arrhythmia independent of focal discharge. Am Heart 1959;58:59-70.

29. Moe GK, Rheinboldt WC, Abildskov JA. A computer model of atrial fibrillation. Am Heart J. 1964;67:200-20

30. Allessie MA Lammers WJEP, Bonke FIM, Hollen SJ. Experimenta Evaluation of Moe's Multiple Wavelet Hypothesis of Atrial Fibrillation. In: Zipes DP and Jalife J (eds), Cardiac Electrophysiology and Arrhythmias, New York; Grune \& Stratton:1985:265-76.

31. Cox JL, Schuessler RB, D'Agostino HJ Jr, et al. The surgical treatment of atrial fibrillation. III. Development of a definitive surgical procedure. J Thorac Cardiovasc Surg. 1991;101: 569-83.

32. Waks JW, Josephson ME. Mechanisms of atrial fibrillation - reentry, rotors and reality. Arrhythm Electrophysiol Rev. 2014;3:90-100.

33. Shiroshita-Takeshita A, Brundel BJ, Nattel S. Atrial fibrillation: basic mechanisms, remodeling and triggers. I Interv Card Electrophysiol. 2005;13:181-93.

34. Allessie MA, Bonke FI, Schopman FJ. Circus movement in rabbit atrial muscle as a mechanism of tachycardia. III. The "leading circle" concept: a new model of circus movement in cardiac tissue without the involvement of an anatomical obstacle. Circ tissue without the

35. Comtois P, Kneller J, Nattel S. Of circles and spirals: bridging the gap between the leading circle and spiral wave concepts of cardiac reentry. Europace. 2005;7(Suppl. 2):10-20.

36. Nattel S. New ideas about atrial fibrillation 50 years on. Nature. 2002;415:219-26

37. Davidenko JM, Kent PF, Chialvo DR, et al. Sustained vortex-like waves in normal isolated ventricular muscle. Proc Nat/ Acad SC USA. 1990;87:8785-9.

38. Pandit SV, Jalife J. Rotors and the dynamics of cardiac fibrillation. Circ Res. 2013:112: 849-62

39. Vaquero M, Calvo D, Jalife J. Cardiac fibrillation: from ion channels to rotors in the human heart. Heart Rhythm. 2008; $5: 872-9$

40. Berenfeld O, Zaitsev AV, Mironov SF, et al. Frequency dependen breakdown of wave propagation into fibrillatory conduction across the pectinate muscle network in the isolated sheep right atrium. Circ Res. 2002;90:1173-80.

41. Schuessler RB, Grayson TM, Bromberg Bl, et al. Cholinergically mediated tachyarrhythmias induced by a single extrastimulus in the isolated canine right atrium. Circ Res. 1992;71:1254-67

42. Mandapati R, Skanes A, Chen J, et al. Stable microreentrant sources as a mechanism of atrial fibrillation in the isolated sheep heart. Circulation. 2000;101:194-9.

43. Mansour M, Mandapati R, Berenfeld O, et al. Left-to-right gradient of atrial frequencies during acute atrial fibrillation in the isolated sheep heart. Circulation. 2001;103:2631-6.

44. Lazar S, Dixit S, Marchlinski FE, et al. Presence of left-to-right atrial frequency gradient in paroxysmal but not persistent atria fibrillation in humans. Circulation. 2004;110:3181-6.

45. Lazar S, Dixit S, Callans DJ, et al. Effect of pulmonary vein isolation on the left-to-right atrial dominant frequency gradient in human atrial fibrillation. Heart Rhythm. 2006;3:889-95.

46. Atienza F Almendral $\rfloor$ Jalife J et al Real-time dominant frequency mapping and ablation of dominant frequency sites in atrial fibrillation with left-to-right frequency gradients predicts long-term maintenance of sinus rhythm. Heart Rhythm. 2009; 6:33-40

47. Narayan SM, Krummen DE, Shivkumar K, et al. Treatment of atrial fibrillation by the ablation of localized sources: CONFIRM (Conventional Ablation for Atrial Fibrillation With or Without Focal Impulse and Rotor Modulation) trial. J Am Coll Cardiol. 2012;60:628-36.

48. Gardner PI, Ursell PC, Fenoglio JJ Jr., et al. Electrophysiologic and anatomic basis for fractionated electrograms recorded from healed myocardial infarcts. Circulation. 1985;72:596-611.

49. de Bakker JM, Wittkampf FH. The pathophysiologic basis of fractionated and complex electrograms and the impact of recording techniques on their detection and interpretation. Circ Arrhythm Electrophysiol. 2010:3.204-13.

50. Katritsis D, Giazitzoglou E, Sougiannis D, et al. Complex fractionated atrial electrograms at anatomic sites of ganglionated plexi in atrial fibrillation. Europace.

51. Hunter RJ, Diab I, Tayebjee M, et al. Characterization of fractionated atrial electrograms critical for maintenance of atrial fibrillation: a randomized, controlled trial of ablation trategies (the CFAE AF trial). Circ Arrhythm Electrophysiol. 2011;4:622-9.

52. Verma A, Mantovan R, Macle $L$, et al. Substrate and trigger ablation for reduction of atrial fibrillation (STAR AF): a andomized, multicentre, international trial. Eur Heart J. 2010;31:1344-56

53. Oral H, Chugh A, Yoshida K, et al. A randomized assessment of the incremental role of ablation of complex fractionated atrial electrograms after antral pulmonary vein isolation fo long-lasting persistent atrial fibrillation. J Am Coll Cardiol. 2009;53:782-9.

54. Li WJ, Bai YY, Zhang HY, et al. Additional ablation of complex fractionated atrial electrograms after pulmonary vein isolation in patients with atrial fibrillation: a meta-analysis. Circ Arrhythm in patients with atrial fibrillation:

55. Wynn GJ, Das M, Bonnett $\perp$, et al. Efficacy of catheter ablation for persistent atrial fibrillation. A systematic review and meta-analysis of evidence from randomized and nonrandomized controlled trials. Circ Arrhythm Electrophysiol. 2014; $7: 841-52$

56. Sharifov OF, Fedorov VV, Beloshapko GG, et al. Roles of adrenergic and cholinergic stimulation in spontaneous atria fibrillation in dogs. J Am Coll Cardiol. 2004;43:483-90.

57. Bettoni M, Zimmermann M. Autonomic tone variations before the onset of paroxysmal atrial fibrillation. Circulation. 2002;105:2753-9.

58. Coumel P. Paroxysmal atrial fibrillation: a disorder of autonomic tone? Eur Heart J. 1994;15(Suppl A):9-16.

59. Armour JA, Murphy DA, Yuan BX, et al. Gross and microscopic anatomy of the human intrinsic cardiac nervous system. Anat Rec. 1997;247:289-98.

60. Vaitkevicius R, Saburkina I, Rysevaite K, et al. Nerve supply of the human pulmonary veins: an anatomical study. Heart Rhythm. 2009;6:221-8.

1. Hou Y, Scherlag BJ, Lin J, et al. Ganglionated plexi modulate extrinsic cardiac autonomic nerve input: effects on sinus rate atrioventricular conduction, refractoriness, and inducibility of atrial fibrillation. J Am Coll Cardiol. 2007;50:61-8.

62. Liu L, Nattel S. Differing sympathetic and vagal effects on atria fibrillation in dogs: role of refractoriness heterogeneity. Am Physiol. 1997;273(2 Pt 2):H805-16.

63. Lim PB, Malcolme-Lawes LC, Stuber T, et al. Intrinsic cardiac autonomic stimulation induces pulmonary vein ectopy and triggers atrial fibrillation in humans. I Cardiovasc Electrophysio. 2011;22:638-46

64. Mao 1 Yin X, Zhang $Y$, et al. Ablation of epicardial ganglionated plexi increases atrial vulnerability to arrhythmias in dogs. Circ Arrhythm Electrophysiol. 2014:7:711-7.

65. Zhou J, Scherlag BJ, Edwards J, et al. Gradients of atria refractoriness and inducibility of atrial fibrillation due to stimulation of ganglionated plexi. J Cardiovasc Electrophysiol. 2007;18:83-90.

66. Scherlag BJ, Yamanashi W, Patel U, et al. Autonomically induced conversion of pulmonary vein focal firing into atrial fibrillation. J Am Coll Cardiol. 2005;45:1878-86.

67. Lu Z, Scherlag BJ, Lin J, et al. Autonomic mechanism for initiation of rapid firing from atria and pulmonary veins: evidence by ablation of ganglionated plexi. Cardiovasc Res. 2009;84:245-52

68. Calkins H, Hindricks G, Cappato R, et al. 2017 HRS/EHRA/ECAS/ APHRS/SOLAECE expert consensus statement on catheter and surgical ablation of atrial fibrillation: Executive summary. $J$ Arrhythm. 2017:33:369-409.

69 Miyazaki S, Kuwahara T, Kobori A, et al. Preprocedura predictors of atrial fibrillation recurrence following pulmonary vein antrum isolation in patients with paroxysmal atria fibrillation: Long-term follow-up results. I Cardiovasc Electrophysiol. 2011;22:621-5.

70. Nanthakumar K, Plumb vj, Epstein AE, et al. Resumption of electrical conduction in previously isolated pulmonary veins: Rationale for a different strategy? Circulation. 2004:109:1226-9.

71. Haïssaguerre M, Hocini M, Sanders P, et al. Catheter ablation of long-lasting persistent atrial fibrillation: Clinical outcome and mechanisms of subsequent arrhythmias. J Cardiovasc Electrophysiol. 2005;16:1138-47.

72. Haïssaguerre $\mathrm{M}$, Sanders $\mathrm{P}$, Hocini $\mathrm{M}$, et al. Catheter ablation of long-lasting persistent atrial fibrillation: Critical structures for termination I Cardiovasc Electrophysiol. 2005:16:1125-37.

73. Schreiber D, Rostock T Frohlich M et al Five-year follow-up after catheter ablation of persistent atrial fibrillation using the stepwise approach and prognostic factors for success. Circ Arrhythm Electrophysiol. 2015;8:308-17.

74. Scherr D, Khairy P, Miyazaki S, et al. Five-year outcome of catheter ablation of persistent atrial fibrillation using termination of atrial fibrillation as a procedural endpoint. Circ Arrhythm Electrophysiol. 2015;8:18-24.

5. Vogler J, Willems S, Sultan A, et al. Pulmonary vein isolation versus defragmentation: The CHASE-AF Clinical Trial. J Am Coll Cardiol. 2015;66:2743-52.

76. Fink $\mathrm{T}$, Schluter $\mathrm{M}$, Heeger $\mathrm{CH}$, et al. Stand-alone pulmonary vein isolation versus pulmonary vein isolation with additional substrate modification as index ablation procedures in patients with persistent and long-standing persistent atrial fibrillation: The Randomized Alster-Lost-AF Trial (Ablation at St. Georg Hospital for Long-Standing Persistent Atrial Fibrillation). Circ Arrhythm Electrophysiol. 2017:10:e005114.

77. Verma A, Jiang CY, Betts TR, et al. Approaches to catheter ablation for persistent atrial fibrillation. N Engl J Med. 2015;372:1812-22

78. Marrouche NF, Wilber D, Hindricks G, et al. Association of atrial tissue fibrosis identified by delayed enhancement MRI and atrial fibrillation catheter ablation. The DECAAF study. JAMA. 2014:311:498-506.

79. Oakes RS, Badger TJ, Kholmovski EG, et al. Detection and quantification of left atrial structural remodeling with delayed-enhancement magnetic resonance imaging in patients with atrial fibrillation. Circulation. 2009:119:1758-67.

80. Rolf S, Kircher S, Arya A, et al. Tailored atrial substrate modification based on low-voltage areas in catheter ablation of atrial fibrillation. Circ Arrhythm Electrophysiol. 2014;7:825-33.

81. Yamaguchi T, Tsuchiya T, Nakahara S, et al. Efficacy of left atria voltage-based catheter ablation of persistent atrial fibrillation. J Cardiovasc Electrophysiol. 2016;27:1055-63.

82. Kottkamp H, Berg J, Bender R, et al. Box Isolation of fibrotic areas (BIFA): A patient-tailored substrate modification approach for ablation of atrial fibrillation. J Cardiovasc Electrophysiol. 2016;27:22-30

83. Chang HY, LO LW, Lin YJ, et al. Long-term outcome of catheter ablation in patients with atrial fibrillation originating from nonpulmonary vein ectopy. I Cardiovasc Electrophysiol. 2013;24:250-8

84. Hayashi $\mathrm{K}$, An Y, Nagashima M, et al. Importance of nonpulmonary vein foci in catheter ablation for paroxysmal atrial fibrillation. Heart Rhythm. 2015;12:1918-24.

85. Santangeli P, Zado ES, Hutchinson MD, et al. Prevalence and distribution of focal triggers in persistent and long-standing persistent atrial fibrillation. Heart Rhythm. 2016;13:374-82.

86. Dixit S, Marchlinski FE, Lin D, et al. Randomized ablation strategies for the treatment of persistent atrial fibrillation: RASTA study. Circ Arrhythm Electrophysiol. 2012:5:287-94.

87. Liang JJ, Elafros MA, Muser D, et al. Pulmonary vein antral isolation and nonpulmonary vein trigger ablation are sufficient to achieve favorable long-term outcomes including transformation to paroxysmal arrhythmias in patients with persistent and long-standing persistent atrial fibrillation. Circ Arrhythm Electrophysiol. 2016:9:e004239.

88. Cutler MJ, Johnson J, Abozguia K, et al. Impact of voltage mapping to guide whether to perform ablation of the posterior wall in patients with persistent atrial fibrillation. J Cardiovasc Electrophysiol. 2016;27:13-21.

89. Bai R, Di Biase L, Mohanty P, et al. Proven isolation of the pulmonary vein antrum with or without left atrial posterio wall isolation in patients with persistent atrial fibrillation. Heart Rhythm. 2016;13:132-40.

90. McLellan AJA, Prabhu S, Voskoboinik A, et al. Isolation of the posterior left atrium for patients with persistent atrial fibrillation: Routine adenosine challenge for dormant posterior left atrial conduction improves long-term outcome. Europace. 2017:19:1958-66.

91. Miller JM, Kowal RC, Swarup V et al. Initial independent outcomes from focal impulse and rotor modulation ablation for atrial fibrillation: Multicenter FIRM registry. J Cardiovasc Electrophysiol. 2014;25:921-9.

92. Buch $E$, Share M, Tung R, et al. Long-term clinical outcomes of focal impulse and rotor modulation for treatment of atrial fibrillation: A multicenter experience. Heart Rhythm. 2016;13:636-41

93. Berntsen RF, Haland TF, Skardal R, et al. Focal impulse and rotor modulation as a stand-alone procedure for the treatment of paroxysmal atrial fibrillation: A within-patient controlled study with implanted cardiac monitoring. Heart Rhythm. 2016;13:1768-74

94. Pappone C, Santinelli V, Manguso F, et al. Pulmonary vein denervation enhances long-term benefit after circumferentia ablation for paroxysmal atrial fibrillation. Circulation. 2004:109:327-34

95. Katritsis DG, Pokushalov E, Romanov A, et al. Autonomic denervation added to pulmonary vein isolation for paroxysmal atrial fibrillation: a randomized clinical trial. J Am Coll Cardio. 2013;62:2318-25

96. Tzou WS, Marchlinski FE, Zado ES, et al. Long-term outcome after successful catheter ablation of atrial fibrillation. Circ Arrhythm Electrophysiol. 2010;3:237-42.

97. Yubing $W$, Yanping $X$, Zhiyu L, et al. Long-term outcome of radiofrequency catheter ablation for persistent atrial fibrillation. Medicine. 2018;97:29-35.

98. Anselmino M, Matta M, D'Ascenzo F, et al. Catheter ablation of atrial fibrillation in patients with left ventricular systolic dysfunction. A systematic review and meta-analysis. Circ Arrhythm Electrophysiol. 2014:7:1011-8.

99. Liang JJ, Callans DJ. Ablation for atrial fibrillation in heart failure with reduced ejection fraction. Cardiac Failure Review. 2018:4:33-7.

100. Zhao DS, Shen Y, Zhang Q, et al. Outcomes of catheter 
105. Jiang RH, Po SS, Tung R, et al. Incidence of pulmonary vein conduction recovery in patients without clinical recurrence after ablation of paroxysmal atrial fibrillation: Mechanistic implications. Heart Rhythm. 2014;11:969-76.

106. Okamatsu H, Okamura K. Strategy and outcome of catheter ablation for persistent atrial fibrillation - impact of progress in the mapping and ablation technologies. Circ J. 2018;82: 2-9.

107. Chang HY, LO LW, Lin YJ, et al. Long-term outcome of cathete ablation in patients with atrial fibrillation originating from the superior vena cava. J Cardiovasc Electrophysio. 2012;23:955-61.

108. Parkash R, Verma A, Tang ASL. Persistent atrial fibrillation: Current approach and controversies. Curr Opin Cardiol. 2010;25:1-7.

109. Brooks AG, Stiles MK, Laborderie J, et al. Outcomes of long-standing persistent atrial fibrillation ablation: A systematic review. Heart Rhythm. 2010:7:835-46.

110. Cheema A, Dong J, Dalal D, et al. Circumferential ablation with pulmonary vein isolation in permanent atrial fibrillation. Am J pulmonary vein isolation
Cardiol. 2007;99:1425-8.

111. Reddy VY, Shah D, Kautzner J, et al. The relationship between contact force and clinical outcome during radiofrequency catheter ablation of atrial fibrillation in the TOCCATA study. Heart Rhythm. 2012;9:1789-95.

112. Itoh T, Kimura M, Tomita H, et al. Reduced residual conduction gaps and favourable outcome in contact force-guided circumferential pulmonary vein isolation. Europace. 2016;18:531-7.

113. Neuzil P, Reddy VY, Kautzner J, et al. Electrical reconnection after pulmonary vein isolation is contingent on contact force during initial treatment: Results from the EFFICAS I study. Circ Arrhythm Electrophysiol. 2013;6:327-33.

114. Neuzil P, Kuck KH, Nakagawa H, et al. Lesion size index for prediction of reconnection risk following RF ablation for PVI. prediction of reconnection risk
Heart Rhythm. 2012;9:55:S492.

115. Das M, Loveday JJ, Wynn GJ, et al. Ablation index, a novel marker of ablation lesion quality: prediction of pulmonary vein reconnection at repeat electrophysiology study and regional differences in target values. Europace. 2016;19: 775-83.

116. Whitaker J, Panikker S, Fastl T, et al. Tissue thickness affects likelihood of acute electrical reconnection at the ostium of the
left atrial appendage following radiofrequency ablation. Pacing left atrial appendage following radiofrequ
Clin Electrophysiol. 2017;40:1218-26.

117. Taghji P, El Haddad M, Phlips T, et al. Evaluation of a strategy aiming to enclose the pulmonary veins with contiguous and optimized radiofrequency lesions in paroxysmal atrial fibrillation: a pilot study. JACC Clin Electrophysio. 2018;4:99-108

118. El Haddad M, Taghji P, Phlips T, et al. Determinants of acute and late pulmonary vein reconnection in contact force-guided pulmonary vein isolation: identifying the weakest link in the ablation chain. Circ Arrhythm Electrophysiol. 2017;10:e004867.

119. Pison L, Tilz R, Jalife J, et al. Pulmonary vein triggers, focal sources, rotors and atrial cardiomyopathy: implications for the choice of the most effective ablation therapy. I Intern Med. 2016:279:449-56.

120. Nattel S, Burstein B, Dobrev D. Atrial remodeling and atria fibrillation: mechanisms and implications. Circ Arrhythm Electrophysiol. 2008;1:62-73.

121. de VoS $C B$, Pisters $R$, Nieuwlaat $R$, et al. Progression from paroxysmal to persistent atrial fibrillation clinical correlates and prognosis. J Am Coll Cardiol. 2010;55:725-31.

122. de Groot NM, Houben RP, Smeets JL, et al. Electropathological substrate of longstanding persistent atrial fibrillation in patients with structural heart disease: epicardial breakthrough. Circulation. 2010;122:1674-82.

123. Santangeli P, Marchlinski FE. Techniques for the provocation, localization, and ablation of non-pulmonary vein triggers for atrial fibrillation. Heart Rhythm. 2017:14:1087-96.

124. Dagres N, Bongiorni MG, Scientific Initiatives Committee, European Heart Rhythm Association, et al. Current ablation European Heart Rhythm Association, et al. Current ablation European Heart Rhythm Association Survey. Europace. 2015;17:1596-600.

125. Tang WH, Lee KT, Tsai WC, et al. The feasibility and correlation of atrial fibrillation vulnerability test to the indices of atrial substrates using atrial burst decremental pacing. Kaohsiung Journal of Medical Sciences. 2013;29:299-303.

126. Berthet K, Lavergne T, Cohen A, et al. Significant association of atrial vulnerability with atrial septal abnormalities in young patients with ischemic stroke of unknown cause. Stroke. 2000;31:398-403

127. Pokushalov E, Romanov A, Shugayev P, et al. Selective ganglionated plexi ablation for paroxysmal atrial fibrillation. Heart Rhythm. 2009;6:1257-64.

128. de Groot N, van der Does L, Yaksh A, et al. Direct proof of endo-epicardial asynchrony of the atrial wall during atrial fibrillation in humans. Circ Arrhythm Electrophysiol. 2016;9:e003648

129. Atienza F, Climent AM, Guillem MS, et al. Frontiers on non-invasive cardiac mapping: Rotors in atrial fibrillation - body surface frequency-phase mapping. Card Electrophysiol Clin. 2015;7:59-69.

130. Salinet $\mathrm{JL}$, Tuan JH, Sandilands AJ, et al. Distinctive patterns of dominant frequency trajectory behavior in drug-refractory persistent atrial fibrillation: Preliminary characterization of spatiotempor 2014;25:371-9.

131. Almeida TP, Chu GS, Bell MJ, et al. The temporal behavior and consistency of bipolar atrial electrograms in human persisten atrial fibrillation. Med Biol Eng Comput. 2018;56:71-83.

132. Salinet J, Schlindwein FS, Stafford P, et al. Propagation of meandering rotors surrounded by areas of high dominan frequency in persistent atrial fibrillation. Heart Rhythm. 2017;14:1269-78.

133. Pappone C, Ciconte G, Vicedomini G, et al. Clinical outcome of electrophysiologically guided ablation for nonparoxysmal atrial fibrillation using a novel real-time 3-dimensional mapping technique: Results from a prospective randomized trial. Circ Arrhythm Electrophysiol. 2018;11:e005904.

134. Honarbakhsh S, Schilling RJ, Providencia R, et al. Automated detection of repetitive focal activations in persistent atrial fibrillation: Validation of a novel detection algorithm and application through panoramic and sequential mapping. J Cardiovasc Electrophysiol. 2018;1-9. 